\section{VTE PROPHYLAXIS FOR PATIENTS IN A SPECIALIST PALLIATIVE CARE CENTRE: A QUALITY IMPROVEMENT PROJECT}

Yuxuan Zhang. Roxburghe House, NHS Grampian

10.1136/spcare-2021-PCC.137

Introduction Venous Thromboembolism (VTE) is a source of significant preventable morbidity and mortality amongst hospital inpatients. NICE recommends considering VTE prophylaxis (VTEp) for patients receiving palliative care. This quality improvement project looked at the completion rate of VTEp assessment at Roxburghe House (RH), a Scottish Specialist Palliative Care Unit, where a VTEp pro forma was first introduced in 2018.

Aim To assess the completion rate of VTEp assessment and decision documentation amongst admissions to RH. To subsequently implement a modified VTEp assessment form to improve VTEp assessment and decision documentation according to NICE guidance.

Methods Admission documents for 36 consecutive patients to RH were reviewed from October to November 2020. Data including primary condition, admission medications, VTEp assessment, and VTEp decisions were recorded. A modified VTEp pro forma in an innovative flow chart format was introduced as the sole intervention. A further 27 consecutive patients' admission documents were subsequently reviewed.

Results 23/36(63.9\%) of all admissions during the initial cycle had VTEp decision documented. 7/36(19.4\%) were for VTEp and 13/36(36.1\%) were not. Only 15/36(41.7\%) had both VTEp assessment and decision documented. Amongst those with no VTEp prescribed nor decision documented, 9/36 (25\%) may benefit from VTEp on review. The VTEp decision documentation rate increased to $23 / 27(85.2 \%)$ after the intervention. 14/27(51.9\%) were for VTEp, 8/27(29.6\%) were not. 23/27(85.2\%) had both VTEp assessment and decision documented. Amongst those with no VTEp prescribed nor decision documented, 3/27(11.1\%) may benefit from VTEp on review. Conclusion Two years after the implementation of VTEp assessment pro forma in RH in 2018, the VTEp assessment completion rate has decreased to pre-2018 level. The redesigned VTEp form has led to an increase in VTEp assessment and decision documentation rate in RH. Further work is required to assess the review of VTEp decisions on an ongoing basis during admission.

\section{Posters 120 - 125 | supportive care}

\section{ARTIFICIAL INTELLIGENCE IN PALLIATIVE CARE: A SYSTEMATIC REVIEW TO IDENTIFY HOW NOVEL DATA ANALYTIC TECHNOLOGIES ARE USED IN THE MANAGEMENT OF PEOPLE WITH SERIOUS ILLNESS}

Osamah Ahmad, Sarah Stanley, Stephen Mason, Amara Nwosu. School of Medicine, University of Liverpool, Marie Curie Hospice Liverpool, Palliative Care Unit, University of Liverpool, International Observatory on End of Life Care, Division of Health Research, Faculty of Health and Medicine, Lancaster University, Liverpool University Hospitals NHS Foundation Trust

\subsection{6/spcare-2021-PCC.138}

Background New technologies, such as artificial intelligence (AI), supported by novel ways of linking and analysing data, are transforming the way that healthcare data is analysed. AI is increasingly being used to support healthcare delivery, and examples of palliative care application are emerging. However, the current scope of (and potential) use of AI in palliative care delivery has not been fully explored. The aim of this project was to define the scope of use of AI methodologies in palliative care studies.

Methods A systematic review of literature was conducted in accordance with the PRISMA guidelines. Four electronic databases were searched, in addition to grey literature searches. AI was used as an umbrella term to include keyword searches for the following: machine learning, deep learning, neutral networks and natural language processing.

Results

Twenty-seven relevant articles were selected The majority of studies described people with cancer $(n=10,37 \%)$, from general palliative $(n=8,30 \%)$ and intensive care populations $(n=4,15 \%)$. Studies using natural language processing were most common $(n=12,44 \%)$, with others mainly utilising machine learning $(n=10,37 \%)$, deep learning $(n=3,11 \%)$ and neural network $(n=2,8 \%)$ methodologies. A variety of outcomes were covered, with most studies predicting survival $(n=8,30 \%)$, identifying goals of care $(n=6,22 \%)$, analysing serious illness conversations $(n=2,9 \%)$ and reporting if palliative care best practice recommendations had been followed in clinical care $(n=2,9 \%)$.

Conclusion Most palliative care AI studies report cancer, use natural language processing and machine learning methods, to predict survival and analyse goals of care. Future studies need to explore how different AI methods can support palliative care, whilst carefully assessing the risks and limitations, to ensure effective use in the management of serious illness.

\section{EFFECT OF HOPE-BASED PROGRAM ON THE HOPE OF PEOPLE WITH PALLIATIVE NEEDS: A RCT PILOT STUDY IN PORTUGAL}

Ana Isabel Querido, Carlos Antonio Laranjeira. School of Health Sciences - Polytechnic of Leiria and cTecCare - Center for Innovative Care and Health Technology, Leiria, Portugal

\subsection{6/spcare-2021-PCC.139}

Background Some studies have demonstrated the effectiveness of interventions in the levels of hope, however the relationship between these interventions and the results is still limited. The aim of the study was to evaluate the efficacy of a hopebased program in nursing on Portuguese adult outpatients with advanced and progressive chronic illness.

Methods A single-blind RCT was conducted to test the hypothesis that outpatients in the intervention group, who participated in the Hope Promotion Program (HPP), have higher levels of hope than those who did not participate in the program. Participants were recruited from Day Hospitals of two medical institutions. Following the individuals' agreement to participate, they were thoroughly screened for eligibility by the main researcher. Inclusion criteria: (1) adults diagnosed with advanced and progressive chronic disease; (2) accompanied by a health care team; and (3) ability to speak and comprehend Portuguese. Hope is measured by the Portuguese version of Herth Hope Index for chronic conditions. The time frames for assessment were at baseline 